\title{
Critical velocity for superfluid flow across the BEC-BCS crossover
}

\author{
D. E. Miller, J. K. Chin, C. A. Stan*, Y. Liu, W. Setiawan, C. Sanner and W. Ketterle ${ }^{\dagger}$ \\ Department of Physics, MIT-Harvard Center for Ultracold Atoms, \\ and Research Laboratory of Electronics, MIT, Cambridge, MA 02139
}

(Dated: October 24, 2018)

\begin{abstract}
Critical velocities have been observed in an ultracold superfluid Fermi gas throughout the BECBCS crossover. A pronounced peak of the critical velocity at unitarity demonstrates that superfluidity is most robust for resonant atomic interactions. Critical velocities were determined from the abrupt onset of dissipation when the velocity of a moving one dimensional optical lattice was varied. The dependence of the critical velocity on lattice depth and on the inhomogeneous density profile was studied.

PACS numbers: 03.75.Kk, 03.75.Lm, 03.75.Ss
\end{abstract}

The recent realization of the BEC-BCS crossover in ultracold atomic gases [1] allows one to study how bosonic superfluidity transforms into fermionic superfluidity. The critical velocity for superfluid flow is determined by the low-lying excitations of the superfluid. For weakly bound fermions, the (Landau) critical velocity is proportional to the binding energy of the pairs, which increases monotonically along the crossover into the BEC regime. However, the speed of sound, which sets the critical velocity for phonon excitations, is almost constant in the BCS regime, but then decreases monotonically on the $\mathrm{BEC}$ side, since the strongly bound molecules are weakly interacting. At the BEC-BCS crossover, one expects a rather narrow transition from a region where excitation of sound limits superfluid flow, to a region where pair breaking dominates. In this transition region, the critical velocity is predicted to reach a maximum [2, 3, ,4]. This makes the critical velocity one of the few quantities which show a pronounced peak across the BEC-BCS crossover in contrast to the chemical potential, the transition temperature [5], the speed of sound [6, 7] and the frequencies of shape oscillations [8], which all vary monotonically.

In this paper, we report the first study of critical velocities across the BEC-BCS crossover, where a Feshbach resonance allows the magnetic tuning of the atomic interactions, and find that superfluid flow is most robust near the resonance. Our observation of a pronounced maximum of the critical velocity is in agreement with the predicted crossover between the two different mechanisms for dissipation.

Critical velocities have been determined before in atomic BECs perturbed by a stirring beam [9, 10, 11] as well as by a 1D moving optical lattice [12]. In both cases, the inhomogeneous density of the harmonically trapped sample had to be carefully accounted for in order to make quantitative comparisons to theory. Here

\footnotetext{
*Present address: Department of Chemistry and Chemical Biology, Harvard University, Cambridge, Massachusetts 02138

${ }^{\dagger}$ Website: cua.mit.edu/ketterle_group
}

we mitigate this problem by probing only the central region of our sample with a tightly focused moving lattice formed from two intersecting laser beams. For decreasing lattice depths, the critical velocity increases and, at very small depths, approaches a value which is in agreement with theoretical predictions.

In our experiments, we first create a superfluid of ${ }^{6} \mathrm{Li}$ pairs according to the procedure described in previous work [13]. Forced evaporative cooling of an even mixture of the two lowest hyperfine states is performed at a magnetic field of $822 \mathrm{G}$, on the BEC side of a broad Feshbach resonance centered at $B_{0}=834 \mathrm{G}$. This results in a nearly pure Bose-Einstein condensate of $N \approx 5 \times 10^{5}$ pairs in a cross optical dipole trap with harmonic trapping frequencies $\nu_{x, y, z}=(65,45,50) \mathrm{Hz}$. The Fermi energy of the system is $E_{F}=h \bar{\nu}(6 N)^{1 / 3}=h \times 7.6 \mathrm{kHz}$. To form the moving lattice, we focus two phase-locked 1064 $\mathrm{nm}$ laser beams to intersect at the sample with an angle of $\sim 90^{\circ}$ (see Figure 1). The resulting 1D lattice has a spatial period of $\lambda_{L}=0.75 \mu \mathrm{m}$. A frequency difference between the two beams of $\Delta \nu$ causes the lattice to move with velocity $v_{L}=\lambda_{L} \Delta \nu$. The beams have $e^{-2}$ waists of $20 \mu \mathrm{m}$ and $60 \mu \mathrm{m}$ respectively, and address a relatively homogeneous region at the center of the cloud which has Thomas-Fermi radii $R_{x, y, z}=(63,91,82) \mu \mathrm{m}$. The minimum density at the position of the $e^{-2}$ waist is $42 \%$ of the central density.

The lattice which necessarily varies in depth across the sample, is characterized by its peak depth $V_{0}$ specified in units of $E_{F}$ or the recoil energy $E_{r}=h^{2} /\left(8 m \lambda_{L}^{2}\right)=$ $h \times 7.3 \mathrm{kHz}$, where $m$ is the molecular mass. The lattice depth is calibrated using Kapitza-Dirac scattering. Due to the inhomogeneity of the lattice, the uncertainty is $40 \%$. The lattice depths explored in this work are sufficiently small such that motion induced in the laboratory frame is negligible, in contrast to [14].

The lattice moving at a constant velocity is adiabatically ramped up and held for a time $t$ up to $2 \mathrm{~s}$, after which the lattice is ramped down and all confinement is switched off. As in previous work [13], a fast magnetic field ramp is used to reduce strong interactions in order 


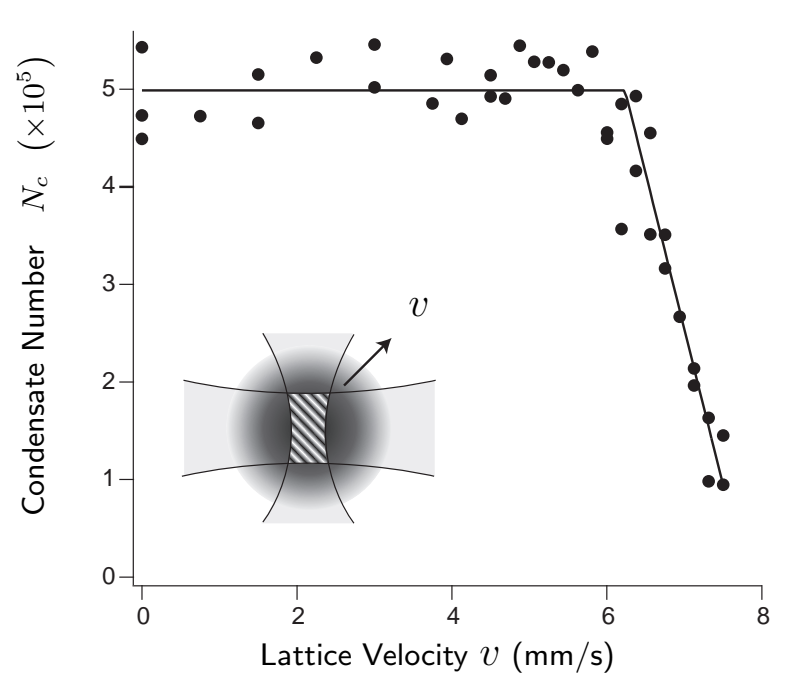

FIG. 1: Onset of dissipation for superfluid fermions in a moving optical lattice. (inset) Schematic of the experiment in which two intersecting laser beams produced a moving optical lattice at the center of an optically trapped cloud (trapping beams not shown). Number of fermion pairs which remained in the condensate $N_{c}$ after being subjected to a $V_{0}=0.2 E_{F}$ deep optical lattice for $500 \mathrm{~ms}$, moving with velocity $v_{L}$, at a magnetic field of $\left.822 \mathrm{G}\left(1 / k_{F} a=0.15\right)\right)$ An abrupt onset of dissipation occurred above a critical velocity $v_{c}$, which we identify from a fit to Equation 1.

to probe the center-of-mass momentum distribution of the pairs. Subsequently, absorption imaging is done on the atomic resonance line at $730 \mathrm{G}$. A bimodal fit reveals the number of pairs remaining in the condensate $N_{c}$, providing a measure of the heating incurred during application of the moving lattice.

Figure 1 illustrates the characteristic dependence of dissipation on the velocity of the moving lattice. At low velocities, the sample is unaffected. Above some critical velocity $v_{c}$, dissipation sets in abruptly. We determine $v_{c}$ from a fit of $N_{c}$ to the intersection of two lines with slopes 0 and $\alpha$ :

$$
N_{\text {cond }}(v)=N_{\text {cond }}(0) \times[1-\max (0, \alpha \times(v-v c))]
$$

The critical velocity which we obtain from this procedure is consistent for a large range of hold times, varying by less than $15 \%$ when the hold time $t$ is changed by a factor of 20. We explore the BEC-BCS crossover by adiabatically ramping the magnetic field to different values after evaporation and repeating the measurement as before. The crossover is parameterized by the interaction parameter $1 / k_{F} a$, where $k_{F}$ is the Fermi wavevector and $a$ is the B-field dependent s-wave scattering length 15 . Again, we observe a threshold for dissipation.

Figure 2 shows the measured critical velocity throughout the BEC-BCS crossover. The maximum near resonance is consistent with the picture of a crossover between two different types of excitation, as discussed in

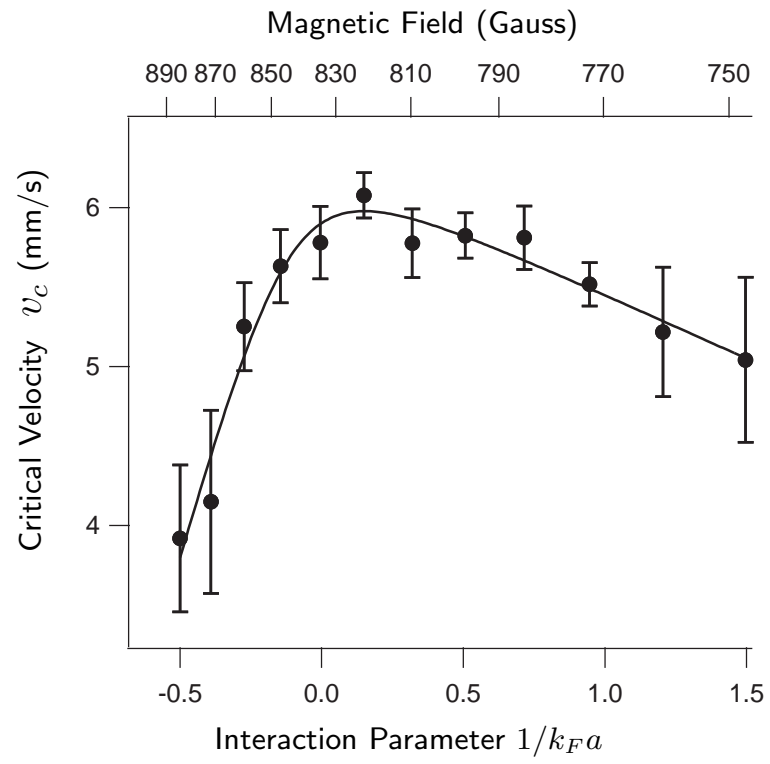

FIG. 2: Critical velocities throughout the BEC-BCS crossover. A pronounced maximum was found at resonance. Data is shown for a $V_{0}=0.2 E_{F}$ deep lattice, held for $\mathrm{t}=500$ $\mathrm{ms}$. The solid line is a guide to the eye.

the introduction, and confirms that superfluidity is most robust on resonance.

To illuminate the role of the inhomogeneous density distribution, we performed experiments in which the entire sample was perturbed by a uniform lattice. Lattice beams with $80 \mu \mathrm{m}$ waists probed a more tightly confined sample of $2 \times 10^{5}$ pairs, with spatial extent $\mathrm{R}_{T F} \simeq 37 \mu \mathrm{m}$. The onset of dissipation seen in Figure 3 is still striking, but now loss is observed at much lower lattice velocities, in spite of a larger Fermi energy $E_{F}=h \times 12.4 \mathrm{kHz}$. Moreover, the onset of dissipation is slightly more gradual. When the magnetic field was varied across the Feshbach resonance, we again found a maximum of the critical velocity near resonance. The lowering of the critical velocity due to the inhomogeneous density profile is expected, since at lower density, both the speed of sound and (on the BCS side) the pairing energy decrease. Although the critical velocity should approach zero in the low density wings of the cloud, we still observe a sudden onset of dissipation at a finite velocity, similar to studies in Ref. [9, 10, 11], where a laser beam pierced through the whole condensate, but in contrast to studies reported in [12].

In the limit of vanishing perturbation, the critical velocity should be given by the Landau criterion. In Figure 4 we address the effects of a finite lattice potential in the original lattice configuration, as depicted in Figure 1. The critical velocity is shown to be a decreasing function of $V_{0}$, saturating in the limit of low lattice depth $\left(V_{0} \leq 0.03 E_{F}\right)$. This behavior is consistent with numerical simulation [4, 16]. Measurements at the smallest lattice depths had large uncertainties, as the hold time required to observe a heating effect of the lattice 


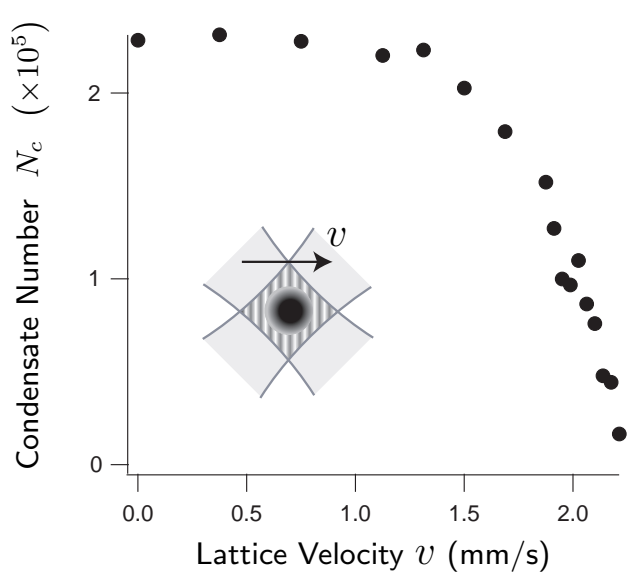

FIG. 3: Effects of density inhomogeneity on the critical velocity. A configuration in which the lattice beams $(80 \mu \mathrm{m})$ were larger than the trapped sample $(37 \mu \mathrm{m})$ results in loss in the condensate number $N_{c}$ at significantly lower velocity. Data is shown for a $V_{0}=0.15 E_{F}$ deep optical lattice held for $200 \mathrm{~ms}$ at a magnetic field of $822 \mathrm{G}$.

approached the natural lifetime of our sample. For this reason we studied the field dependence (Figure 2) at an intermediate lattice depth, where $v_{c}$ was more well defined.

For comparison with theory we reference the local Fermi velocity at the trap center $v_{F}=v_{F}^{\prime}(1+\beta)^{-1 / 4}$ $=39 \mathrm{~mm} / \mathrm{s}$, where $v_{F}^{\prime}=\sqrt{2 E_{F} / m}$ is the Fermi velocity of a non-interacting gas at the trap center, and $\beta=-0.58$ is a universal parameter characterizing unitarity limited interactions [17, 18, 19]. For vanishing lattice depth, the observed critical velocity at unitarity approaches $v_{c} / v_{F}=0.25$. If we use the local Fermi velocity $v_{F, w}$ at the $e^{-2}$ waist of the lattice, we obtain $v_{c} / v_{F, w}=0.34$. The difference between these values indicates the uncertainty due to residual density inhomogeneity. The local speed of sound in a Fermi gas at unitarity is

$$
c_{s}=v_{F, l o c}(1+\beta)^{1 / 2} / \sqrt{3}=0.37 v_{F, l o c} .
$$

The critical velocity for pair breaking is

$$
v_{\text {pair }}=\left(\left(\sqrt{\Delta^{2}+\mu^{2}}-\mu\right) / m\right)^{1 / 2}=0.34 v_{F, l o c}
$$

with $\Delta=0.50 v_{F, l o c}^{2} / 2 m[17,19]$ and $\mu=(1+\beta) v_{F, l o c}^{2} / 2 m$. These two values should provide approximate upper bounds to the critical velocity at unitarity [2, 3]. It seems natural that the combination of both excitation mechanisms lowers the critical velocity further. Within these uncertainties, and those of the density, the theoretical predictions agree with the experimental results.

Up until now, we have deferred a discussion of how the moving lattice couples to the excitations. In a pure system at zero temperature, one would expect the excitation spectrum to exhibit discrete resonances, where the perturbation couples only to modes with the $k$-vector of the

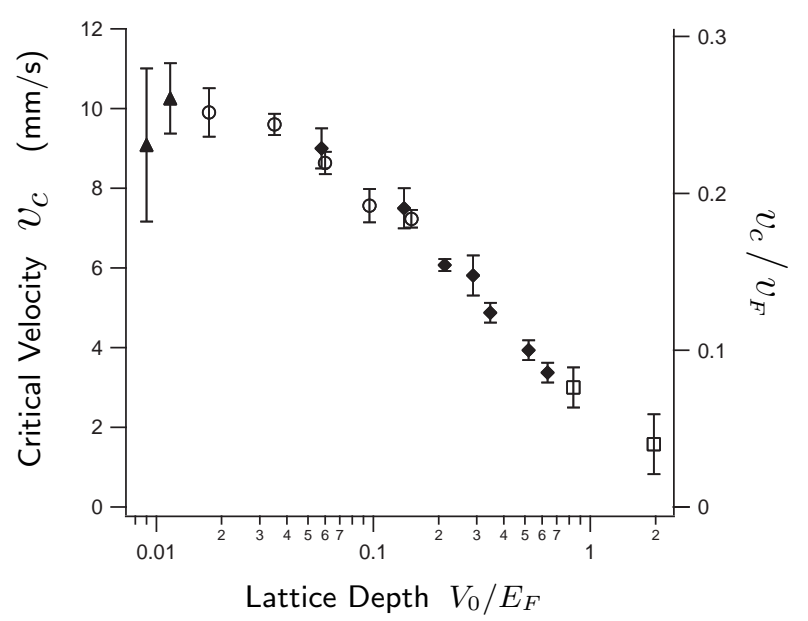

FIG. 4: Critical velocities at different lattice depths. The results show $v_{c}$ to be a decreasing function of lattice depth $V_{0}$. In the limit of low $V_{0}, v_{c}$ converges to a maximum value of 0.25 $v_{F}$. Data was taken near resonance, at $822 \mathrm{G}\left(1 / k_{F} a=0.15\right)$ for hold times t $=250 \mathrm{~ms}, 500 \mathrm{~ms}, 1000 \mathrm{~ms}, 2000 \mathrm{~ms}$ (squares, diamonds, circles, triangles).

lattice. On the other hand, at finite temperature, it is possible that the lattice drags along thermal atoms which are point-like perturbations and can create excitations at all $k$-vectors. Our observation that the dissipation sets in at a certain threshold velocity and increases monotonically with velocity is consistent with the participation of the thermal component.

We further elucidated the role of thermal excitations, by varying the temperature. Gradually reducing the trap depth from $U_{0}$ to $U_{m i n}$, during exposure to a lattice moving above $v_{c}$, will suppress the accumulation of a thermal component. The lifetime in this case exceeded that for a sample held at a fixed depth of either $U_{0}$ or $U_{\text {min }}$. For Bose-Einstein condensates, theoretical papers emphasized the role of the thermal component in the Landau damping process in a moving lattice [20, 21]. This was confirmed qualitatively in an experiment at Florence [12] in which the lifetime of the sample was drastically improved by eliminating the thermal atoms.

In our experiments, the clouds heated up during the exposure to the moving lattice. Figure 5 shows the increase in the number of thermal atoms and the loss in the total number of atoms due to evaporative cooling. In an idealized model, where density is fixed, constant dissipation would result in a linear decrease in the number of atoms due to evaporative cooling. Our data show an accelerated decrease, possibly reflecting increased dissipation due to the increasing fraction of thermal atoms. However, an accurate model should include the change in density (and therefore critical velocity) during the exposure time. Additional impurity atoms (e.g. sodium atoms) could cause dissipation even at zero temperature and would allow more controlled studies of the dissipa- 


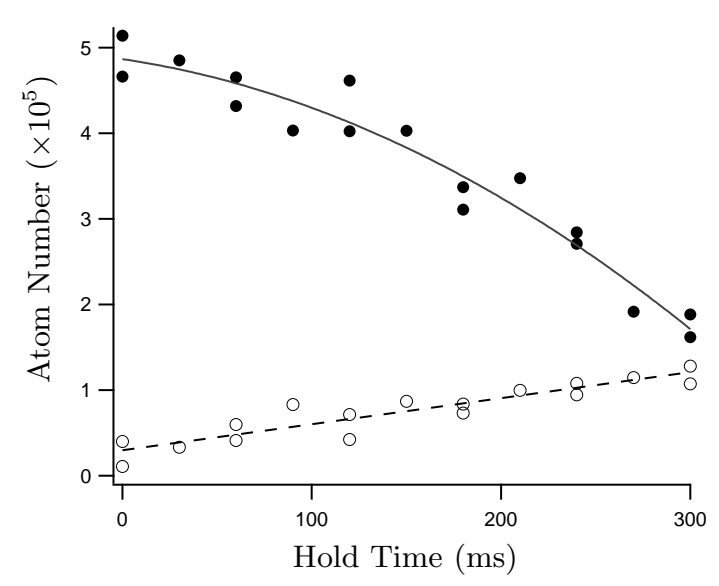

FIG. 5: Number of pairs which remained in the condensate $N_{c}$ (filled circles) and thermal component $N_{t h}$ (open circles) after being held in a $V_{0}=0.35 E_{F}$ deep optical lattice moving with velocity $v_{L}=6 \mathrm{~mm} / \mathrm{s}$ for a variable hold time. The thermal component shows a linear increase (dashed line), whereas $N_{\text {cond }}$ showed an accelerated loss, and is fit to a quadratic function (solid line).

tion mechanism. Unpaired atoms in clouds with population imbalance may not play this role because of phase separation effects [22].

Another possible dissipation mechanism in a lattice is the creation of two excitations through a dynamical or modulational instability. Such an instability [23] occurs already for weakly interacting particles moving through a lattice with momentum $q$, when they collide and scatter into states with momenta $q \pm \delta q$, analogous to optical parametric generation 24]. This process is energetically possible only above $0.5 q_{B}$, where $q_{B}=h / 2 \lambda_{L}$ is the Bragg momentum which defines the edge of the Brillouin zone. This corresponds to a velocity $v=11 \mathrm{~mm} / \mathrm{s}$ for fermion pairs (and twice this value for single atoms). Since the highest critical velocities we observe are slightly below this threshold, and strongly decrease already for relatively small $V_{0}$, it is very unlikely, that dynamical instabilities play a role in our experiments. Moreover, such instabilities should be strongly modified by Pauli blocking. For our ratio of local Fermi momentum to the Bragg momentum of 0.9, the first band is nearly full in the center of the cloud. For Bose-Einstein condensates, it has been recently predicted [25] and experimentally shown [14] that strong interactions can lower the threshold for the dynamical instability, close to the Mottinsulator transition. The range of 1-D lattice depths explored here $\left(V_{0} \leq 2 E_{r}\right)$ is far from the 1D Mott-insulator regime. We have observed the loss of coherence which typically accompanies the superfluid to Mott insulation transition to occur only beyond $V_{0} \simeq 25 E_{r}$.

In conclusion, we have used a novel optical lattice geometry to determine critical velocities in the BEC-BCS crossover without the complications of strong density inhomogeneity. This configuration could be applied to studies in atomic Bose gases which so far have been limited by the inhomogeneous density [9, 10, 11, 12]. In addition, it would be interesting to study dynamical instabilities for fermions and the role of Pauli blocking. The authors would like to thank Aviv Keshet for experimental assistance. This research has been supported by the NSF and the Office of Naval Research.

[1] S. Giorgini, L. P. Pitaevskii, and S. Stringari, preprint condmat/0706.3360

[2] R. Sensarma, M. Randeria, and T.-L. Ho, Phys. Rev. Lett. 96, 090403 (2006).

[3] R. Combescot, M. Y. Kagan, and S. Stringari, Phys. Rev. A 74, 042717 (2006).

[4] A. Spuntarelli, P. Pieri, and G. C. Strinati, pre-print /condmat/0705.2658 .

[5] C. A. R. Sá de Melo, M. Randeria, and J. R. Engelbrecht, Phys. Rev. Lett. 71, 3202 (1993).

[6] R. Haussmann, W. Rantner, S. Cerrito, and W. Zwerger, Phys. Rev. A 75, 023610 (2007).

[7] J. Joseph et al., Phys. Rev. Lett. 98, 170401 (2007).

[8] A. Altmeyer et al., Phys. Rev. Lett. 98, 040401 (2007) 98, 040401 (2007).

[9] C. Raman et al., Phys. Rev. Lett. 83, 2502 (1999).

[10] R. Onofrio et al., Phys. Rev. Lett. 85, 2228 (2000).

[11] C. Raman et al., J. Low Temp. Phys. 122, 99 (2001).

[12] L. De Sarlo et al., Phys. Rev. A 72, 013603 (2005).

[13] J. Chin et al., Nature 443, 961 (2006).

[14] J. Mun et al., preprint condmat/0706.3946.

[15] M. Bartenstein et al., Phys. Rev. Lett. 94, 103201 (2005).

[16] S. Ianeselli, C. Menotti, and A. Smerzi, J. Phys. B 39, S135 (2006).

[17] J. Carlson, S.-Y. Chang, V. R. Pandharipande, and K. E. Schmidt, Phys. Rev. Lett. 91, 050401 (2003).

[18] G. E. Astrakharchik, J. Boronat, J. Casulleras, and S. Giorgini, Phys. Rev. Lett. 93, 200404 (2004).

[19] J. Carlson and S. Reddy, Phys. Rev. Lett. 95, 060401 (2005).

[20] S. Tsuchiya and A. Griffin, Phys. Rev. A 70, 023611 (2004).

[21] S. Konabe and T. Nikuni, J. Phys. B 39, S101 (2005).

[22] Y. Shin et al., Phys. Rev. Lett. 97, 030401 (2006).

[23] B. Wu and Q. Niu, Phys. Rev. A 64, 061603 (2001).

[24] G. K. Campbell et al., Phys. Rev. Lett. 96, 020406 (2006).

[25] E. Altman et al., Phys. Rev. Lett. 95, 020402 (2005). 